\title{
Interface Profiles near Three-Phase Contact Lines in Electric Fields
}

\author{
Juergen Buehrle, Stephan Herminghaus, and Frieder Mugele* \\ University of Ulm, Applied Physics Department, D-89069 Ulm, Germany
}

(Received 14 March 2003; published 18 August 2003)

\begin{abstract}
Long-range electrostatic fields deform the surface profile of a conductive liquid in the vicinity of the contact line. We have investigated the equilibrium profiles by balancing electrostatic and capillary forces locally at the liquid vapor interface. Numerical results show that the contact angle at the contact line approaches Young's angle. Simultaneously, the local curvature displays a weak algebraic divergence. Furthermore, we present an asymptotic analytical model, which confirms these results and elucidates the scaling behavior of the profile close to the contact line.
\end{abstract}

DOI: 10.1103/PhysRevLett.91.086101

PACS numbers: 68.03.-g, 47.65.+a, 68.05.-n

The rapid developments in biotechnology and combinatorial chemistry create an increasing demand for microfluidic devices that allow for precise control and manipulation of tiny amounts of liquids [1]. Recently, new strategies have been developed for open microfluidic systems, i.e., systems that are characterized by the presence of solid-liquid-vapor or solid-liquid-liquid triple lines [2,3]. In this approach, manipulation of liquids relies on the presence of gradients in interfacial tensions that are switchable via an external control parameter. Electric fields are arguably the most interesting choice as a control parameter, because of their flexibility, high switching rates, and little power consumption. In particular, the electrowetting effect attracts considerable attention [3-8]. Electrowetting amounts to the fact that the contact angle of partially wetting conductive liquids on insulating substrates can be reduced by applying a voltage $U$ between the liquid and a counterelectrode on the substrate, which is covered by a thin insulating layer [cf. Fig. 1(a)]. If combined with suitably patterned counterelectrodes, net forces can be exerted in order to move liquid droplets on the surface.

Electrowetting is well understood as long as the applied voltage is low. On a macroscopic scale, i.e., sufficiently far away from the contact line, the voltage dependence of the contact angle is given by the so-called Lippmann equation, e.g., [5],

$$
\cos \theta_{L}=\cos \theta_{Y}+\eta,
$$

where $\theta_{L}$ is the Lippmann contact angle and $\theta_{Y}$ is Young's contact angle. $\eta=\varepsilon_{0} \varepsilon_{r} U^{2} /(2 \gamma d)$ is a dimensionless number representing the ratio of electrostatic and capillary forces. $\gamma$ is the surface tension of the liquid. $\varepsilon_{r}$ and $d$ are the dielectric constant and the thickness of the insulating layer, respectively. $\varepsilon_{0}$ is the electric susceptibility of vacuum. At high voltage, however, the standard model of electrowetting fails. Instead of decreasing to zero as predicted theoretically, the contact angle is found to saturate typically around $30^{\circ}$, thereby limiting the maximum force that can be generated in microfluidic or other applications. A variety of mechanisms were proposed in order to explain contact angle saturation [6-8]. Most of them attribute a crucial role to the divergence of electric fields close to the wedge-shaped edge of the droplets. However, a deeper understanding of these saturation effects has been hampered thus far, because the geometry of

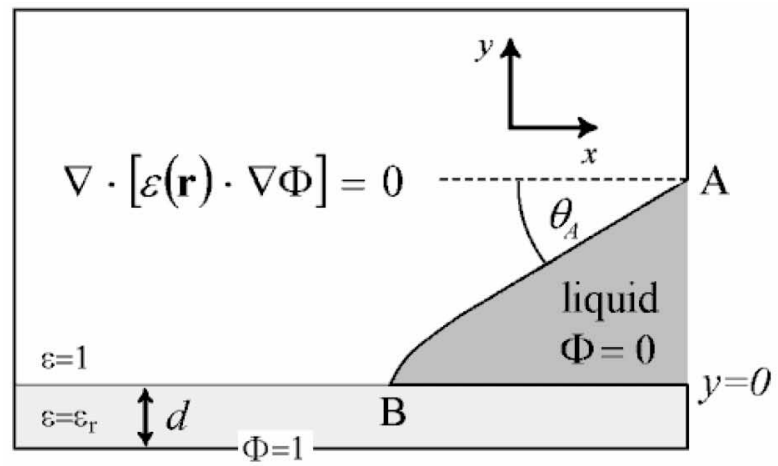

(a)

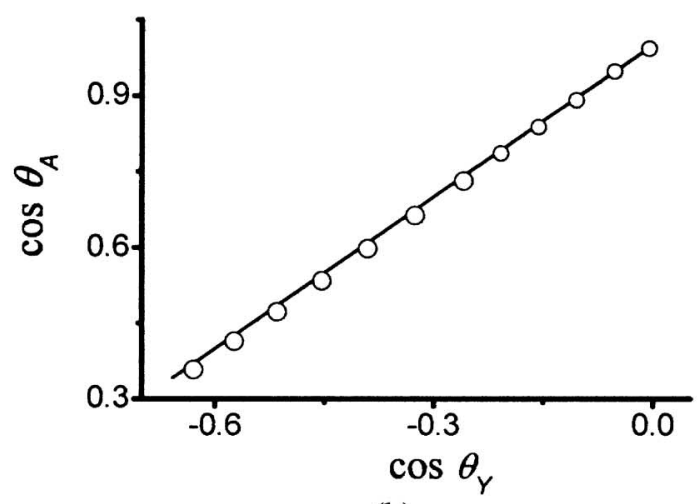

(b)

FIG. 1. (a) Geometrical setup. Unless stated otherwise, length and height of the box were 80. See text for further details. (b) $\cos \left(\theta_{A}\right)$ vs $\cos \left(\theta_{Y}\right)$. Symbols: numerical results (for the five smallest values of $\theta_{A}$, the box size was increased to 500). Solid line: prediction of Eq. (1), assuming $\theta_{A}=\theta_{L}$. 
the liquid surface in the vicinity of the contact line was not known [6,7].

In the present Letter, we present a new numerical approach that allows us to calculate in a self-consistent manner both the field distribution and the equilibrium surface profiles close to the contact line. We find that the contact angle approaches Young's angle in the immediate vicinity of the substrate, even at high voltage when the Lippmann angle is small. At the same time, the surface curvature diverges algebraically with an exponent $-1<$ $\nu<0$. The numerical results are explained by an asymptotic analytical model.

The geometry of our system is shown in Fig. 1(a). The liquid, which is translationally invariant in one direction, is connected to a reservoir at zero pressure. In the following, all length scales will be normalized to the thickness $d$ of the insulator. The electrical potential is $\Phi=0$ both for the liquid and on the top of the simulation box. At the bottom electrode, the dimensionless electrical potential $\Phi=1$ is applied (normalized by the applied voltage $U$ ). On the left-hand edge of the box, Dirichlet boundary conditions were specified with $\Phi$ corresponding to an infinite parallel plate capacitor partially filled with a dielectric. Point A was kept fixed throughout the calculations at a height of 40 above the substrate, whereas the contact line (point B) was allowed to relax. In order to calculate the equilibrium surface profile, we followed an iterative procedure. Initially, we began with a straight liquid vapor interface with a slope corresponding to the asymptotic angle $\theta_{A}$. For this geometry, the field distribution was calculated by solving numerically the equation $\nabla \cdot[\varepsilon(\mathbf{r}) \cdot \nabla \Phi]=0$ (where $\varepsilon=1$ in the gas phase and $\varepsilon=\varepsilon_{r}$ in the insulating layer) outside the liquid with the boundary conditions as specified above [9]. Since the electric field $\mathbf{E}=-\nabla \Phi$ vanishes inside the liquid, the Maxwell stress acting on the surface is given by $\Pi_{e l}=$ $\varepsilon_{0} \mathbf{E}^{2} / 2$. In mechanical equilibrium, this stress must be balanced by the capillary pressure $\Pi_{\text {cap }}=\gamma \kappa$, where $\kappa$ is the local curvature of the surface. If we describe the surface profile by a function $x=f(y)$, mechanical equilibrium requires that

$$
-\frac{f^{\prime \prime}(y)}{\left(1+f^{\prime}(y)^{2}\right)^{3 / 2}}=\frac{\eta}{\varepsilon_{r}}(\nabla \Phi)^{2} .
$$

This equation can be obtained from a variational minimization of the free energy of the system [10]. Equation (2) was integrated numerically using $\Phi$ as obtained above, with the initial conditions that the profile emerges in point $\mathrm{A}$ with a slope corresponding to $\theta_{A}$. Thus, we obtained a new (curved) profile $f(y)$, which was used again to calculate $\Phi$ via $\nabla \cdot[\varepsilon(\mathbf{r}) \cdot \nabla \Phi]=0$ for the next iteration step. Good convergence was obtained after typically ten iterations. Unless stated otherwise, we used $\varepsilon_{r}=1$. For other values of $\varepsilon_{r}$, qualitatively similar results were obtained.
Any profile obtained from this procedure represents the mechanical equilibrium for a given set of parameters $\varepsilon_{r}$, $\eta$, and $\theta_{A}$. In order to relate $\theta_{A}$ to Young's angle $\theta_{Y}$ for a given system, we calculated the free energy $F$, which, in units of $\gamma d^{2}$, is given by

$$
\begin{aligned}
F\left(\varepsilon_{r}, \eta, \theta_{A}\right)= & -\cos \left(\theta_{Y}\right) L+\int_{A}^{B} d S-\frac{\eta}{\varepsilon_{r}} \int_{V_{1}} d V(\nabla \Phi)^{2} \\
& -\eta \int_{V_{2}} d V(\nabla \Phi)^{2}
\end{aligned}
$$

Here, $L$ is the normalized length of the solid-liquid interface. The second term on the right-hand side gives the contour length of the mechanically equilibrated surface profile $f(y)$. The last two terms represent the electrostatic field energies in the gas phase $\left(V_{1}\right)$ and inside the dielectric layer $\left(V_{2}\right)$, respectively. The thermodynamic equilibrium configuration is calculated by minimizing $F$ with respect to $\theta_{A}$. As a result, we found that $\theta_{Y}$ and the equilibrium value of $\theta_{A}$ are indeed related via Eq. (1); i.e., $\theta_{A}=\theta_{L}$ [see Fig. 1(b)]. The fact that the Lippmann equation is recovered as a result of the numerical procedure confirms the accuracy of the calculations. We point out that the Lippmann relation was found to hold within the numerical accuracy down to the smallest angles investigated $\left(\theta_{A, \min }=\theta_{L, \min } \approx 5^{\circ}\right)$; i.e., we find no indication for electrostatically induced contact angle saturation at high voltage. This result is in contrast to previously published conclusions, which were based on a simplified model [7].

In the following, we will focus on the behavior of the surface profile in the vicinity of the contact line. Figure 2(a) shows a series of profiles for various values of $\eta$. Obviously, their curvature in the vicinity of the contact line increases with increasing $\eta$. In order to minimize numerical errors, $\theta_{A}$ was kept constant throughout these calculations. This implies that each profile corresponds to a different value of $\theta_{Y}$. In order to investigate the asymptotic behavior of the surface profiles in more detail, we define the normalized derivative

$$
\chi=\frac{f^{\prime}(y)-\cot \theta_{Y}}{\cot \theta_{A}-\cot \theta_{Y}} .
$$

By virtue of this definition, $\chi \rightarrow 1$ for $f^{\prime}(y) \rightarrow \cot \left(\theta_{A}\right)=$ $\cot \left(\theta_{L}\right)$, and $\chi \rightarrow 0$ for $f^{\prime}(y) \rightarrow \cot \left(\theta_{Y}\right)$. Figure 2(b) shows $\chi$ for the same data as in Fig. 2(a). Far away from the contact line, $\chi \rightarrow 1$ for all the profiles, due to the boundary condition at point A. Because of the long range nature of the electrostatic forces, the profiles approach the Lippmann angle only very slowly-in fact algebraically, as we will see below. Therefore, there is no well-defined height above which the Lippmann angle is reached. For practical purposes, however, deviations become negligible for $y \gg 1$. Close to the contact line (i.e., for $y \rightarrow 0$ ), $\chi$ approaches zero for all the profiles. The 


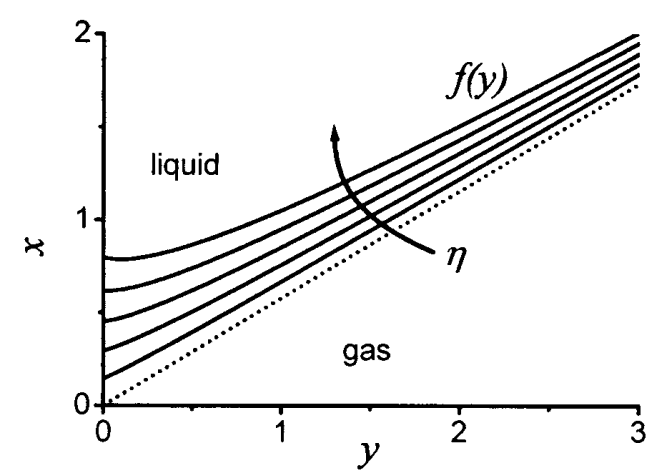

(a)

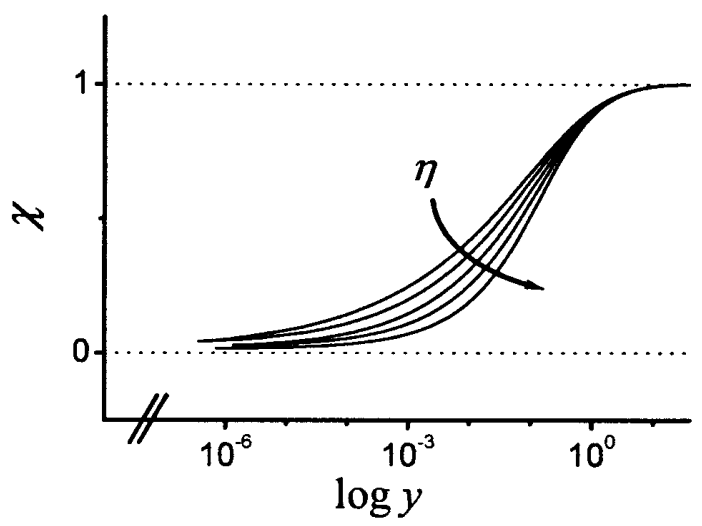

(b)

FIG. 2. (a) Equilibrium surface profiles. $\left(\theta_{A}=60^{\circ} ; \eta=0.2\right.$, $\left.0.4, \ldots, 1.0 ; \varepsilon_{r}=1\right)$. (b) Normalized slope $\chi$ of the surface profiles vs height [parameters as in (a)]. Note the logarithmic scale on the abscissa. The dashed horizontal lines at $\chi=0$ and at $\chi=1$ correspond to $\theta_{Y}$ and $\theta_{A}$, respectively.

asymptotic contact angle at the contact line in electrowetting is thus given by Young's angle $\theta_{Y}$, independent of both the applied voltage and $\varepsilon_{r}$. This suggests that there is no electrostatic force acting at the contact line, although the electric field is expected to diverge close to the contact line.

In order to understand this finding on a more fundamental basis, we investigated the asymptotic behavior of the electrostatic pressure. A logarithmic plot of $\left.(\nabla \Phi)^{2}\right|_{x=f(y)}$ [Fig. 3(a)] shows that $\Pi_{e l}$ diverges algebraically for $y \rightarrow 0$; i.e., $\Pi_{e l} \propto y^{\nu}$. The exponent $\nu$ decreases slightly with increasing $\eta$. In all our calculations, we found $-1<\nu<0$. The squares and triangles in Fig. 3(b) represent the values $\nu$ for $\varepsilon_{r}=1$ and $\varepsilon_{r}=2$, respectively.

The origin of this algebraic behavior for $y \ll 1$ can also be understood analytically. On a length scale much smaller than 1, the geometry can be approached by a perfectly conductive wedge with an opening angle $\alpha$ on an infinitely thick dielectric layer (below, we will also recover analytically that $\alpha=\theta_{Y}$ ). For this geometry, the Laplace equation can be solved analytically in polar coordinates $r, \varphi$. If the wedge occupies the range $2 \pi-$ $\alpha<\varphi<2 \pi$, we have $\varepsilon=\varepsilon_{r}$ for $0<\varphi<\pi$ and $\varepsilon=1$ for $\pi<\varphi<2 \pi-\alpha$. The boundary conditions are $\Phi=$ 0 for $\varphi=0$ and for $\varphi=2 \pi-\alpha$. Furthermore, $D_{y}$ and $E_{x}$ are continuous for $\varphi=\pi$ (with $\mathbf{D}=\varepsilon_{0} \varepsilon \mathbf{E}$ being the dielectric displacement). The solutions of the Laplace equation can be decomposed into eigenmodes $\Phi_{n}=$ $r^{\mu_{n}} \sin \left(\mu_{n} \varphi\right)$, where $\mu_{n}$ must satisfy the condition

$$
\varepsilon_{r} \tan \left[\mu_{n}(\pi-\alpha)\right]+\tan \left(\mu_{n} \pi\right)=0 .
$$

For the special case $\varepsilon_{r}=1$, this simplifies to $\sin \left[\mu_{n}(2 \pi-\alpha)\right]=0$ or $\mu_{n}=n \pi /(2 \pi-\alpha)$, as found in standard textbooks [11]. For $r \rightarrow 0$, the mode with $n=$ 1 dominates [12]. It is easily seen that $\frac{1}{2}<\mu_{1}<1$ for physically meaningful values of $\varepsilon_{r}$ and $\alpha$. Since $\Pi_{e l} \propto$ $(\nabla \Phi)^{2}$, we recover $\Pi_{e l} \propto r^{\nu}$, with $\nu=2\left(\mu_{1}-1\right)$, i.e., $-1<\nu<0$ as obtained before numerically. The dashed lines in Fig. 3(b) represent numerical solutions of Eq. (5) (note that there is no fitting parameter). Thus, both the electric field as well as the electric pressure diverge algebraically. However, the divergence is rather weak.

Since the surface profiles obey Eq. (2), we also have $f^{\prime \prime} \propto r^{\nu}$ and thus $f^{\prime} \propto$ const $+r^{\nu+1}$ for $r \rightarrow 0$, with $0<\nu+1<1$. Hence, the curvature of the profiles diverges while the asymptotic slope (and thus $\alpha$ ) remains finite [13].

The above results imply that the divergence of $\Pi_{e l}$ is weaker than a delta function. Under these conditions, a rigorous variational calculation shows that the local contact angle $\alpha$ should indeed be equal to Young's angle [14], as found above numerically [Fig. 2(b)]. This conclusion may also be verified explicitly by analyzing the force balance at the contact line. The electrostatic force $F_{e l}$ acting on the contact line can be found by integrating $\Pi_{e l}$ over some finite range $\delta r$ along the surface profile, and then considering the limit $\delta r \rightarrow 0$. Hence,

$$
F_{e l} \propto \lim _{\delta r \rightarrow 0} \int_{0}^{\delta r} \Pi_{e l}(y) d y .
$$

Since $\Pi_{e l}$ scales as $\delta r^{\nu}$, we have $F_{e l} \propto \delta r^{\nu+1}$. Since $\nu+$ $1>0, F_{e l}$ vanishes for $\delta r \rightarrow 0$; i.e., the electrostatic force does not contribute to the force balance at the contact line. The only forces acting on the contact line are the interfacial tensions. Therefore, $\alpha$ must indeed be equal to $\theta_{Y}$.

These results should also be of interest to the dynamics of electrowetting. In the absence of electric fields, there are two mechanisms affecting the dynamics of (partial) wetting: viscous dissipation inside the liquid droplet and frictional dissipation at the moving contact line [15]. For volatile liquids, such as water, it was also suggested that local evaporation and condensation processes affect the contact line dynamics [16]. It is widely accepted that the value of the contact angle, at the contact line, has a strong influence on the friction of the moving contact line. In the case of dynamic electrowetting, earlier studies assumed that the geometry close to the contact line is given by a straight wedge, with an opening angle equal to $\theta_{L}$ [17]. 


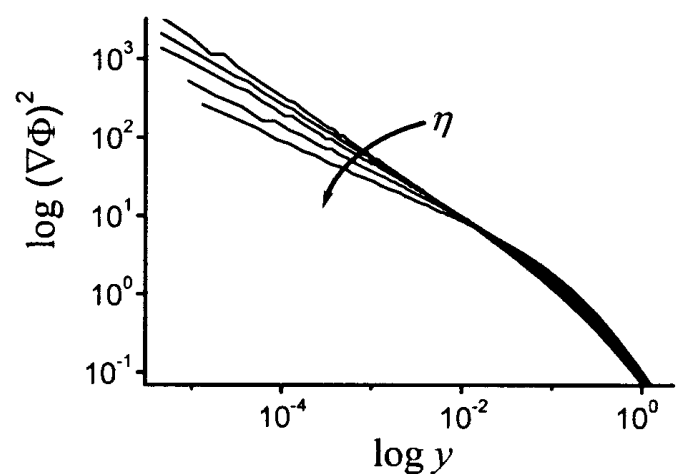

(a)

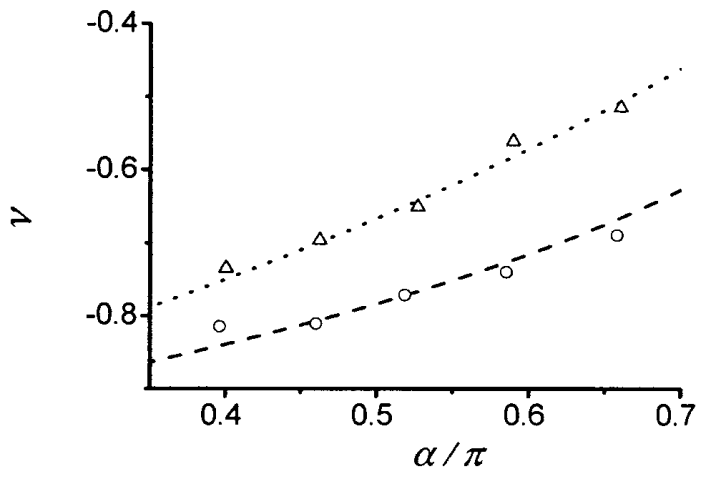

(b)

FIG. 3. (a) $(\nabla \Phi)^{2} \propto \Pi_{e l}$ vs height. Note the algebraic behavior for $y \rightarrow 0$. (b) Exponent $\nu$ of $\Pi_{e l}$ for various values of $\alpha=\theta_{Y}$. Triangles: numerical results for $\varepsilon_{r}=1$; circles: numerical results for $\varepsilon_{r}=2$. Dotted and dashed lines: values corresponding to the solutions of Eq. (5) for $\varepsilon_{r}=1$ and $\varepsilon_{r}=2$, respectively.

As we showed above, neither of these assumptions is true. It will be interesting to study the corrections that arise if the actual equilibrium geometry is incorporated into these dynamic models.

We thank M. Schulz for fruitful discussions and comments. This work was funded by the German Science Foundation within the priority program "Wetting and Structure Formation at Interfaces."

*Corresponding author.

Email address: frieder.mugele@physik.uni-ulm.de
[1] A. Manz and R.C. Anderson, in Topics in Current Chemistry (Springer, Berlin, 1998), Vol. 194; G. M. Whitesides and A. D. Stroock, Phys. Today 54, 42 (2001).

[2] H. Gau, S. Herminghaus, P. Lenz, and R. Lipowsky, Science 283, 46 (1999); B. S. Gallardo, V. K. Gupta, F. D. Eagerton, L. I. Jong, V.S. Craig, R. R. Shah, and N. L. Abbott, Science 283, 57 (1999); D.W. Kataoka and S. M. Troian, Nature (London) 402, 794 (1999); A. A. Darhuber, J. P. Valentino, J. M. Davis, and S. M. Troian, Appl. Phys. Lett. 82, 657 (2003).

[3] M. G. Pollack and R. B. Fair, Appl. Phys. Lett. 77, 1725 (2000); T. B. Jones, M. Gunji, M. Washizu, and M. J. Feldman, J. Appl. Phys. 89, 1441 (2001); F. Mugele and S. Herminghaus, Appl. Phys. Lett. 81, 2303 (2002).

[4] M.W. J. Prins, W. J. J. Welters, and J.W. Weekamp, Science 291, 277 (2001).

[5] C. Quilliet and B. Berge, Curr. Opin. Colloid Interface Sci. 6, 1 (2001).

[6] M. Vallet, M. Vallade, and B. Berge, Eur. Phys. J. B 11, 583 (1999).

[7] K. H. Kang, Langmuir 18, 10318 (2002).

[8] V. Peykov, A. Quinn, and J. Ralston, Colloid Polym. Sci. 278, 789 (2000); H. J. J. Verheijen and M.W. J. Prins, Langmuir 15, 6616 (1999).

[9] We used a two-dimensional finite element code implemented in the partial differential equation toolbox of the software package MATLAB (The MathWorks Inc., Natick, MA, U.S.A.).

[10] T. Chou, Phys. Rev. Lett. 87, 106101 (2001).

[11] L. D. Landau and E. M. Lifschitz, Lehrbuch der Theoretischen Physik VIII (Akademie-Verlag, Berlin, 1976).

[12] This result is consistent with the asymptotic behavior found in Refs. [6,7] for opening angle $\alpha$.

[13] It is interesting to note that a similar situation was also found for vapor bubbles spreading on solid surfaces in heat exchangers at the so-called "boiling crisis" transition. V.S. Nikolayev and D. A. Beysens, Europhys. Lett. 47, 345 (1999).

[14] R. Finn, A Series of Comprehensive Studies in Mathematics (Springer-Verlag, New York, 1986), p. 284.

[15] P. G. DeGennes, Rev. Mod. Phys. 57, 827 (1985); J. DeConinck, M. J. DeRuijter, and M. Voué , Curr. Opin. Colloid Interface Sci. 6, 49 (2001).

[16] M. E. R. Shanahan, Langmuir 17, 8229 (2001).

[17] T. D. Blake, A. Clarke, and E. H. Stattersfield, Langmuir 16, 2928 (2000); C. Decamps and J. DeConinck, Langmuir 16, 10150 (2000). 